\title{
Comment on: Burden of central nervous system complications in sickle cell disease: A systematic review and meta-analysis
}

\author{
R. Grant Steen ${ }^{1}$ \\ ${ }^{1}$ Louisiana State University Health Sciences Center
}

January 31, 2022

Comment on: Burden of central nervous system complications in sickle cell disease: A systematic review and meta-analysis

R. Grant Steen, $P h D$

103 Van Doren Place

Chapel Hill, NC 27517

G_Steen_MediCC@yahoo.com

Dear Editor;

I read with interest a recent paper in Pediatric Blood $\&$ Cancer(1), about cognitive impairment in children with sickle cell disease (SCD). I'm always grateful to have my work cited, especially since our paper is almost 20 years old (2).

However, it seems that Lee et al . have gone to great lengths to mischaracterize our work. They write (pg. 5) that, "SCI [silent cerebral infarct] and absence of SCI/CVA [cerebrovascular accident] were verified by review of brain MRIs in all studies except Ghafuri et al . and Steen et al . In these studies, absence of SCI/CVA was defined as no mention of cerebral infarcts in the subject's medical records." Also on pg. 5, they write that, "An alternative analysis was conducted excluding studies with insufficient definition of overt stroke and/or SCI based on clinical opinion (Ghafuriet al . and Steen et al .)". I cannot speak for Ghafuriet al, but we did a careful review of MRI findings for every single patient in our study, as is necessary for a paper in theAmerican Journal of Neuroradiology. This should be pellucidly clear because the title of our paper is, "Cognitive Impairment in Children with Hemoglobin SS Sickle Cell Disease: Relationship to MR Imaging Findings and Hematocrit". All our SCD patients were evaluated by at least two neuroradiologists, and patients thought to be abnormal were evaluated by a third neuroradiologist blinded as to previous findings (2). One wonders what other errors may be present in Leeet al (1).

Fortunately, this mischaracterization of our work did not lead to incorrect conclusions (1). A comparison of Table 1 to Supplementary Table 6, in which our paper and Ghafuri et al were deleted from analysis, shows that none of the major findings were changed much by deleting these papers (1). I agree with most conclusions in Lee et al .; in fact, I would go further. In uncited work from St. Jude Children's Research Hospital, we found that children with SCD can suffer cognitive impairment even in the absence of MRI evidence of brain injury (3). We tested a hypothesis that children with SCD who are completely normal by MRI can still be cognitively impaired, as predicted by a model of diffuse brain injury. A total of 54 patients with hemoglobin SS (average age 10.9 years \pm 2.9 years SD) were examined with the Wechsler Intelligence Scale for Children-III (WISC-III) and were randomly matched by age, race, and gender to healthy children from the Wechsler normative database. Patients were also imaged at 1.5 Tesla with standard imaging sequences. Among 30 patients judged normal by MRI, there were substantial deficits in Wechsler Full-Scale IQ, Verbal 
IQ, and Performance IQ (all P < 0.01), when compared to African-American controls. The patient Wechsler Full-Scale IQ was 12.9 points lower than that of matched controls and decreased as a function of age $(\mathrm{P}=$ 0.014). These findings strongly suggest that there is diffuse brain injury in SCD patients, perhaps associated with chronic hypoxia (3).

\section{References}

1. Lee S, Lucas S, et al . Burden of central nervous system complications in sickle cell disease: A systematic review and meta-analysis. 2022. Pediatr Blood Cancer . 2022 Jan 17:e29493.

2. Steen RG, Miles MA, et al . Cognitive impairment in children with hemoglobin SS sickle cell disease: relationship to MR imaging findings and hematocrit. 2003. AJNR Am J Neuroradiol . 24(3):382-9.

3. Steen RG, Fineberg-Buchner C, et al . Cognitive deficits in children with sickle cell disease. 2005. J Child Neurol . 2005 Feb;20(2):102-7. 